\title{
Is there a role for pregabalin as premedication in pediatric anesthesia?
}

\author{
Jessica Nghiem ${ }^{1} \cdot$ Stephen C. Brown ${ }^{1} \cdot$ Kazuyoshi Aoyama ${ }^{1,2}(1)$ \\ Received: 6 March 2021 / Accepted: 30 March 2021 / Published online: 9 April 2021 \\ (c) Japanese Society of Anesthesiologists 2021
}

Keywords Pediatrics $\cdot$ Pregabalin $\cdot$ Preanesthetic Medication

Premedication is commonly prescribed in pediatric anesthesia for the anxious, distressed or poorly compliant child who may also have developmental or behavioral issues or a history of unpleasant experiences in healthcare settings. Children in heightened states of anxiety are likely to present a challenge for separating from caregivers, poorly tolerate induction of anesthesia and show postoperative psychological or maladaptive behaviors. An ideal premedication agent would achieve anxiolysis and sedation with safe and easy administration, have a predictable onset time and have minimal adverse effects. Less commonly studied benefits to premedication include sparing effects of anesthesia and analgesia requirements to facilitate recovery.

Pregabalin is a structural analogue of $\gamma$-aminobutyric acid, which was developed as an anticonvulsant agent and is now extensively used in the realm of adult pain therapy. Pregabalin's mechanism of action involves its binding to the $\alpha_{2} \delta$ subunits of voltage-gated calcium channels, inhibiting calcium influx and attenuating release of excitatory neurotransmitters within the central nervous system [1]. Comparative studies with gabapentin in adults have demonstrated pharmacokinetic similarities with negligible metabolism in humans; no inhibition of hepatic enzyme systems and plasma clearance by renal excretion [1]. When administered orally, pregabalin has better bioavailability and is absorbed more rapidly than gabapentin, with maximum plasma concentrations attained within $1 \mathrm{~h}$ compared to $3-4 \mathrm{~h}$ for gabapentin $[1,2]$.

Kazuyoshi Aoyama

kazu.aoyama@utoronto.ca

1 Department of Anesthesia and Pain Medicine, The Hospital for Sick Children, 555 University Ave, \#2211, Toronto, ON M5G 1X8, Canada

2 Program in Child Health Evaluative Sciences, SickKids Research Institute, Toronto, Canada
Evidence from adult studies suggests the predominant effects of pregabalin are antinociceptive, anticonvulsive and anxiolytic and it is indicated for management of persistent pain or neuropathic pain [1]. Although generally well tolerated, dizziness and somnolence are the most commonly reported adverse effects of pregabalin [2]. In theory, pregabalin's desired effects of anxiolysis, antinociception and somnolence are advantageous in its use as premedication in pediatric anesthesia, but there is a question about the existence of good quality evidence to support this use.

The perioperative use of pregabalin has not been widely studied in the pediatric population. The majority of evidence supporting the current perioperative use of pregabalin in children is derived from studies investigating the safety and efficacy of gabapentanoids for the treatment of neuropathic pain or seizures in children or as premedication in the adult population.

A recent 2019 systematic review of the safety and efficacy of gabapentin and pregabalin for pain in children identified a sparsity of evidence supporting the clinical use of gabapentinoids as analgesic agents in children [3]. Out of the seven studies included in the qualitative synthesis, only one study investigated the efficacy of pregabalin, specifically in the context of fibromyalgia. In contrast to the extensive evidence in favor of the safety and analgesic efficacy of pregabalin in adults, this systematic review highlights the need for more high-quality studies in the pediatric population [4]. In practice, certain institutions with specialized pediatric pain services continue to recommend gabapentanoids as a nonopioid adjunct option used for the treatment of neuropathic pain in children, with pregabalin as second line therapy to gabapentin [5]. Brown et al. in their 2016 study, comparing gabapentin with amitriptyline for complex regional pain syndrome type I and neuropathic pain in children, did show both drugs to be effective in reducing pain [6]. Thus, gabapentin can be indicated as a non-opioid pain adjunct for specific cases in children. 
The safety and tolerability of pregabalin as an adjunctive therapy in children with refractory partial seizures was evaluated by Mann et al [7]. The randomized, placebocontrolled, escalating-dose and multiple-dose study demonstrated favorable safety and tolerability in patients aged 1 month to 16 years at doses up to and including $10 \mathrm{mg} / \mathrm{kg} /$ day, with higher doses up to $15 \mathrm{mg} / \mathrm{kg} /$ day well tolerated in children aged 1 month to 6 years. Similar to studies in adults, dizziness and somnolence were reported as the most common adverse effects from pregabalin. Although this study provides guidance on safe and maximum dosing of pregabalin as an anticonvulsant in children, the therapeutic dose for preoperative anxiolysis or sedation remains undetermined.

White et al. conducted a dose-ranging study to investigate the effect of pregabalin on preoperative anxiety and sedation levels in adult participants undergoing minor elective surgery [8]. The findings, measured by the patient-reported Verbal Rating Scale (VRS), demonstrated that preoperative pregabalin administration (75-300 mg PO) increased preoperative sedation in a dose-related fashion, but did not demonstrate correspondingly reduced preoperative anxiety, postoperative pain or improve recovery process. The anxiolytic and sedative effects of pregabalin were also studied in a small randomized controlled trial of 25 children aged 4-6 years old with dental anxiety undergoing dental treatment [9]. Eskandarian et al. reported the mean changes of Visual Analogue Scale (VAS) Anxiety and Sedation scores from baseline to $2 \mathrm{~h}$ post-dose were statistically significantly greater in the group premedicated with pregabalin $75 \mathrm{mg}$ compared to placebo. However, in practice, $2 \mathrm{~h}$ is an impractically long onset-time for the purpose of premedication in what is often a time-pressured or dynamic environment of pediatric anesthesia.

A recently published randomized, double-blinded, controlled study by Talaat et al. investigated the use of pregabalin $(5 \mathrm{mg} / \mathrm{kg})$ compared to midazolam $(0.75 \mathrm{mg} / \mathrm{kg})$ as premedication in pediatric day-case surgery-adding to the small existing body of evidence of pregabalin use in the pediatric perioperative setting [10]. The authors reported on the sparing effects of intraoperative anesthetic and analgesic requirements as the primary outcomes. With respect to the primary outcomes, the pregabalin group resulted in significantly lower mean rank fentanyl (17.32 vs $43.68 \mu \mathrm{g}$, $p<0.001)$ and sevoflurane consumption $(1.6 \pm 0.4$ vs $2.6 \pm 0.3 \%, p<0.001)$. The analgesic-sparing results favoring pregabalin compared with midazolam suggest there is a potential role for pregabalin in acute surgical pain in pediatric cases. This translated clinically in the statistically significantly lower FLACC pain scales in the pregabalin group up to $8 \mathrm{~h}$ post-operatively. Interestingly, there were no significant differences found between the two groups when comparing the preoperative modified Ramsey Sedation Score before and after $30 \mathrm{~min}$ of premedication.
The quest to find the ideal premedication in pediatric anesthesia that would consistently allay anxiety or provide adequate sedation to facilitate smooth induction of anesthesia with optimal safety and recovery profile is an ongoing pursuit. Although there are theoretical advantages of pregabalin use in this context, there is a paucity of studies investigating the efficacy and dosage of pregabalin in children and only small scale randomized controlled trials in pediatric anesthesia currently exist, which have yet to provide consistent or convincing evidence. This state of evidence in this field leaves scope for future, larger scale studies to investigate the advantages of pregabalin as a premedication agent with antinociceptive benefits. Pregabalin and gabapentin have similar pharmacokinetic properties, but they also differ in onset, duration, and interactions with other medications (e.g., NSAIDs). Hence, a potential future trial is a comparative trial between pregabalin, gabapentin, and a well-established comparator agent (e.g., midazolam). As such, one can distinguish whether a particular gabapentinoid is more effective than the other and the common agent in pediatric cohorts as a preoperative non-opioid analgesic and anxiolytic adjuvant.

Acknowledgements As a recipient of Research Commendation, 20212023 Merit Awards Competition, Department of Anesthesiology and Pain Medicine, University of Toronto, KA acknowledges Department of Anesthesiology and Pain Medicine, University of Toronto, and Department of Anesthesia and Pain Medicine, Hospital for Sick Children, for their effort to secure academic time to conduct the current work.

Author contribution JN and KA conceived this paper and wrote the first draft of the manuscript. All authors wrote subsequent iterations of the manuscript and approved the final manuscript.

Funding Not applicable.

\section{Declarations}

Conflict of interest The authors declare that they have no competing interests related to this publication.

\section{References}

1. Bockbrader HN, Wesche D, Miller R, Chapel S, Janiczek N, Burger P. A comparison of the pharmacokinetics and pharmacodynamics of pregabalin and gabapentin. Clin Pharmacokinet. 2010;49(10):661-9.

2. Schmidt PC, Ruchelli G, Mackey SC, Carroll IR. Perioperative gabapentinoids: choice of agent, dose, timing, and effects on chronic postsurgical pain. Anesthesiology. 2013;119(5):1215-21.

3. Egunsola O, Wylie CE, Chitty KM, Buckley NA. Systematic review of the efficacy and safety of gabapentin and pregabalin for pain in children and adolescents. Anesth Analg. 2019;128(4):811-9. 
4. Derry S, Bell RF, Straube S, Wiffen PJ, Aldington D, Moore RA. Pregabalin for neuropathic pain in adults. Cochrane Database of Systematic Reviews. 2019;1(1):CD007076 https://doi.org/10. 1002/14651858.CD007076.pub3

5. Gai N, Naser B, Hanley J, Peliowski A, Hayes J, Aoyama K. A practical guide to acute pain management in children. J Anesth. 2020;34(3):421-33.

6. Brown S, Johnston B, Amaria K, Watkins J, Campbell F, Pehora $\mathrm{C}, \mathrm{McGrath} \mathrm{P}$. A randomized controlled trial of amitriptyline versus gabapentin for complex regional pain syndrome type I and neuropathic pain in children. Scand J Pain. 2016;13:156-63.

7. Mann D, Liu J, Chew ML, Bockbrader H, Alvey CW, Zegarac E, Pellock J, Pitman VW. Safety, tolerability, and pharmacokinetics of pregabalin in children with refractory partial seizures: a phase I, randomized controlled study. Epilepsia. 2014;55(12):1934-43.

8. White PF, Tufanogullari B, Taylor J, Klein K. The effect of pregabalin on preoperative anxiety and sedation levels: a dose-ranging study. Anesth Analg. 2009;108(4):1140-5.
9. Eskandarian T, Eftekharian H, Soleymanzade R. Efficacy and safety of premedication with single dose of oral pregabalin in children with dental anxiety: a randomized double-blind placebo-controlled crossover clinical trial. Dent Res J (Isfahan). 2015;12(6):528-33.

10. Talaat SM, El-Gendy HA. Effect of pregabalin versus midazolam premedication on the anesthetic and analgesic requirements in pediatric day-case surgery: a randomized controlled trial. Egypt J Anaesth. 2021;37(1):50-6.

Publisher's Note Springer Nature remains neutral with regard to jurisdictional claims in published maps and institutional affiliations. 